\title{
Nouvelle-Calédonie. Terre de corail de Pascale JOANNOT
}

\author{
Isabelle Leblic
}

\section{OpenEdition \\ Journals}

Édition électronique

URL : http://journals.openedition.org/jso/5975

DOI : $10.4000 /$ jso.5975

ISSN : $1760-7256$

Éditeur

Société des océanistes

\section{Édition imprimée}

Date de publication : 15 décembre 2009

Pagination : 329-330

ISBN : 978-2-85430-026-0

ISSN : 0300-953x

Référence électronique

Isabelle Leblic, « Nouvelle-Calédonie. Terre de corail de Pascale JoAnnot », Journal de la Société des

Océanistes [En ligne], 129 | juillet-décembre 2009, mis en ligne le 15 décembre 2009, consulté le 24 septembre 2020. URL : http://journals.openedition.org/jso/5975 ; DOI : https://doi.org/10.4000/jso 5975

(c) Tous droits réservés 


\section{COMPTES RENDUS}

JoAnnot Pascale, 2008. Nouvelle-Calédonie. Terre de corail, Paris, Éditions Maison de la NouvelleCalédonie/Solaris, 123 p., bibliogr., ill. couleur.

Cet ouvrage a été publié à l'occasion de l'exposition du même nom qui a été inaugurée à l'Aquarium de la Porte Dorée à Paris en décembre 2008 et que nous avons annoncée dans le Jso Spécial environnement paru fin 2008. Depuis, cette exposition, réalisée par la Maison de Nouvelle-Calédonie (MNC) dans le cadre de l'inscription le 7 juillet 2008 d'une partie des lagons de Nouvelle-Calédonie, de sa diversité récifale et des écosystèmes associés, au patrimoine mondial de l'UNESCO, circule de ville en ville ${ }^{1}$.

«Depuis juillet 2008, $15000 \mathrm{~km}^{2}$ de lagons et récifs calédoniens répartis en six sites sont reconnus et "labellisés" patrimoine mondial de l'UNESCO. » (p. 16)

L'auteur de cet ouvrage est le commissaire de l'exposition conçue à partir d'une idée originale du directeur de l'agence Trans-Faire de Paris, Virginio Gaudenzi. La visite de cette exposition m'a laissé un excellent souvenir ; dommage de n'avoir reçu ce catalogue qu'en mai 2009, ne me permettant pas de le mettre en écho avec cette visite.

L'avant-propos, intitulé « L'homme est inséparable de la nature », est signé par le président du gouvernement de Nouvelle-Calédonie, Harold Martin, et des présidents des trois provinces, Paul Néaoutyine pour le Nord, Philippe Gomès pour le Sud et Neko Hnepeune pour les Îles. Dans la droite ligne des accords de Matignon et de Nouméa qui fêtent respectivement leurs vingtième et dixième anniversaires, cette réalisation éco-conçue ${ }^{2}$ - une première en France - entend célébrer le destin commun des hommes en harmonie avec leur nature :

« Ensemble nous partageons la certitude que le développement de notre pays ne peut se faire qu'en conciliant l'économie et l'écologie. Au moment où se réalisent de nouvelles usines métallurgiques dans les provinces Nord et Sud de la Nouvelle-Calédonie, nous devons affirmer notre engagement de mieux connaître, préserver et protéger ce patrimoine qui nous accueille depuis des millénaires.[...] Car l'homme est inséparable de la nature qu'il ne peut dominer durablement sans la respecter. » (p. 7)
Après une très courte introduction de Joël Viratelle (directeur de la MNC), Virginio Gaudenzi (réalisateur de l'exposition) et Pascale Joannot (commissaire de l'exposition) insistant sur le besoin de partager « la beauté de ce bien naturel à "caractère exceptionnel, universel et inestimable" que sont les récifs coralliens de Nouvelle-Calédonie » (p. 8), viennent six chapitres faisant écho aux divers volets de l'exposition et aux six parties de la structure du corail. Ils déclinent la Nouvelle-Calédonie en « Terre océanienne » (pp. 1017), « Terre de corail» (pp. 18-35), « Terre nature» (pp. 36-59), « Terre des hommes » (pp. 60-83), « Terre de paradoxe» (pp. 84-97) et «Terre de partage» (pp. 98-113). L'ouvrage se termine sur les remerciements d'usage (pp. 114-115), la présentation de cinq des photographes ayant illustré le parcours (pp. 116120), et une bibliographie (pp. 122-123).

Le premier chapitre nous fait aborder cette Terre océanienne par huit belles photographies aériennes montrant la diversité des littoraux calédoniens et de leurs couleurs : blanc de l'écume, bleu des lagons, vert de la végétation et rouge et ocre de la terre minière. Il invite au voyage et à la découverte de cette terre entourée de «l'un des ensembles coralliens les plus remarquables de la planète », et de ses populations très attachées à ce « bien commun à respecter et à partager, d'une importance majeure aux plans coutumier, culturel, scientifique, économique et touristique » (p. 11). Deux cartes complètent cette présentation. Afin de présenter les récifs coralliens du monde, la première situe la Nouvelle-Calédonie sur un planisphère, en signalant particulièrement les « récifs coralliens de l'outre-mer français » et en indiquant l'importance de chaque zone économique exclusive (ZEE) et la surface des formations coralliennes. La Nouvelle-Calédonie arrive ici en première position en termes d'importance des formations coralliennes avec $40000 \mathrm{~km}^{2}$ (80\% de l'ensemble corallien des collectivités d'outre-mer) et une longueur de récif supérieure à $2000 \mathrm{~km}$, pour $18564 \mathrm{~km}^{2}$ de terres émergées et $740000 \mathrm{~km}^{2}$ de ZEE (soit cinq fois celle de la France métropolitaine et $15,8 \%$ de la ZEE totale française).

«Les récifs coralliens de Nouvelle-Calédonie représentent un des ensembles coralliens les plus remarquables de la planète tant par sa structure que par la diversité biologique dont il permet le développement. » (p. 16)

1. Du 22 juin au 4 octobre 2009 à Nice.

2. «C'est une exposition qui se soucie de l'environnement, sur le fond et dans la forme » et elle « sera la première à afficher son bilan carbone » mesuré selon la méthode de l'ADEME, pour « rendre compte de la cohérence environnementale de notre démarche par un indice incontestable afin d'éviter "l'auto proclamation verte" trop couramment répandue. » (p. 111). 
Les auteurs rapportent les données présentées à l'échelle de la France métropolitaine pour permettre au visiteur de se rendre compte de son importance. Au niveau mondial, les récifs coralliens sont les «plus grandes bio-constructions de la planète » et sont situés en zone intertropicale : $284300 \mathrm{~km}^{2}$ (à peine plus de la moitié de la France) offrant un potentiel économique et une source de subsistance à plus de 500 millions de personnes (dix fois la population métropolitaine et $8 \%$ de la population mondiale) dans une centaine de pays bordés par ces récifs. L'outre-mer français place la France au quatrième rang mondial des grandes régions coralliennes avec $14280 \mathrm{~km}^{2}$ de récifs coralliens, derrière l'Indonésie $\left(51020 \mathrm{~km}^{2}\right.$ de récifs), l'Australie $\left(48960 \mathrm{~km}^{2}\right)$ et les Philippines $\left(25060 \mathrm{~km}^{2}\right)$. La seconde carte, figurant la NouvelleCalédonie et les îles, avec ses découpages provincial et communal, accompagne une présentation géographique de cet archipel du bout du monde (à $18368 \mathrm{~km}$ de Paris) et de ses populations : 244600 habitants en 2008 dont les Kanak, le peuple d'origine, représentent $44,1 \%$ à côté de $34,1 \%$ d'Européens, $9 \%$ de Wallisiens et Futuniens, 2,6\% de Tahitiens, 2,5\% d'Indonésiens, $1,4 \%$ de Ni-Vanuatu et $1,4 \%$ de Vietnamiens, $0,4 \%$ de Chinois et 4,3\% d'autres communautés dont des Japonais.... (selon l'ISEE).

Le second chapitre nous fait entrer dans le vif du sujet : la terre de corail déclinée en plusieurs souschapitres (" "Mégalocorallis" ", "Architectes de talent », « Quelle est la nature du corail : animal, végétal ou minéral?», « Du macrocosme au microcosme », "Coraux fluorescents », " La NouvelleCalédonie honorée par la science »). Extrêmement bien illustré et documenté par de belles photographies et des schémas très pédagogiques, il permet au néophyte de tout savoir sur le corail - voir, par exemple, la présentation de neuf espèces de coraux multiformes avec leur nom commun, leur nom scientifique et une photographie couleur (p. 23) ; l'arbre de vie schématique du corail : les Cnidaire descendent, dans l'ordre du vivant, des animaux (Métazoaires) qui, avec les champignons et les plantes, proviennent des Eucaryotes avec les Achées et les Bactéries (p. 25) ; ou encore le schéma de la structure d'une colonie de corail (p. 28).

Le troisième chapitre présente les différents écosystèmes («natures ») calédoniens: les $4000 \mathrm{~km}^{2}$ de forêt humide ( $21 \%$ du territoire) face aux $350 \mathrm{~km}^{2}$ de forêt sèche, les $4400 \mathrm{~km}^{2}$ de maquis minier (23\% de la superficie !) et les $6000 \mathrm{~km}^{2}$ de savane (40\%). Puis l'auteur fait percevoir l'importance des espèces endémiques de la forêt humide, autrement dit celles qui n'existent nulle part ailleurs, en nous offrant un aperçu de quelques-unes d'entre elles: les roussettes et chauves-souris, seuls mammifères installés en Nouvelle-Calédonie avant l'arrivée de l'homme, dont six espèces sur les neuf actuelles sont endémiques, parmi lesquelles la roussette rousse, Pteropus ornatus... et la fougère arborescente, Cyathea intermedia. Puis, à la rencontre de la mer et de la terre se trouve la célèbre mangrove calédonienne - qui ne connaît pas le « cœur de Voh » qui s'étale sur $200 \mathrm{~km}^{2}$ - et les non moins célèbres crabes de palétuviers, Scylla serrata, dont on apprend tout du cycle de reproduction... De nombreuses photos présentent la biodiversité des lagons et récifs de Nouvelle-Calédonie et leurs étonnants habitants.

Le quatrième chapitre permet de découvrir les hommes qui peuplent ce territoire et leur histoire, à savoir les migrations au moyen de grandes pirogues, puis l'arrivée des Européens sur de grands navires. Là encore, on passe de la mer à la terre avec la découverte de «l'or vert» que fut la garniérite découverte par Jules Garnier et est encore le nickel. L'auteur présente enfin en quelques pages la coutume kanak (pp. 70-79).

Les deux derniers chapitres abordent des questions actuelles de la Nouvelle-Calédonie : les paradoxes climatiques et naturels tout comme écologiques et humains, et le partage actuel de toute cette richesse via le classement à l'unESCO. Ainsi, on apprend que « les coraux sont d'excellents indicateurs climatiques » et que « l'enregistrement des changements du climat se fait à l'échelle de la colonie corallienne et à celle du récif » (p. 86) en vertu de leur « mémoire d'éléphant» et de leur «fragilité de cristal» (p. 85). Contraintes naturelles, changements climatiques et pressions anthropiques entraînent des destructions totales ou partielles de ce milieu et «ont des conséquences pour l'homme en termes de santé publique, d'économie et de société » (p. 95). Parmi les risques les plus célèbres, on pense évidemment à la gratte, ou ciguatera, qui se développe sur les coraux morts et dont le responsable est la micro-algue Gambierdiscus toxicus consommée par les poissons herbivores et qui entre dans la chaîne alimentaire jusqu'à l'homme via les poissons carnivores.

Voilà donc un beau petit ouvrage, très pédagogique et accessible au plus grand nombre, non spécialiste de ces questions, qui permet à tous de se familiariser avec la Nouvelle-Calédonie et sa biodiversité, tant marine que terrestre.

Isabelle LEBLIC, CNRS-LACITO, Villejuif

\section{MichalON Thierry, 2009. L'outre-mer français. Évolu- tion institutionnelle et affirmations identitaires, Paris, L'Harmattan, coll. Grale, 162 p.}

Dans ce court ouvrage, l'auteur développe deux idées-force, dans une perspective qu'il qualifie d' « anthropologie juridique » (p. 15) : d'une part, il met au jour un brouillage juridique mettant fin à l'opposition entre la «République intra-nationale » (France métropolitaine et départements d'outre-mer) soumise au droit commun et la «République extranationale » que constituaient les territoires d'outremer (devenus collectivités sui generis), aux règles de droit distinctes de celles imposées par l'autorité centrale. D'autre part, il montre le caractère semblable des outre-mers, autant sur le plan économique que sur celui des relations ambiguës avec la France, malgré une diversité de statuts censée refléter la diversité des situations locales. Chacune de ces thèses est reprise respectivement dans les deux parties de l'ouvrage, ellesmêmes subdivisées en quatre et cinq chapitres. 